\title{
Commentarii Mathematici Helvetici
}

Knus, M.-A. / Ojanguren, M. / Parimala, R.

Positive-definite quadratic bundles over the plane.

Commentarii Mathematici Helvetici, Vol.57 (1982)

PDF erstellt am: Dec 16, 2008

\section{Nutzungsbedingungen}

Mit dem Zugriff auf den vorliegenden Inhalt gelten die Nutzungsbedingungen als akzeptiert. Die angebotenen Dokumente stehen für nicht-kommerzielle Zwecke in Lehre, Forschung und für die private Nutzung frei zur Verfügung. Einzelne Dateien oder Ausdrucke aus diesem Angebot können zusammen mit diesen Nutzungsbedingungen und unter deren Einhaltung weitergegeben werden. Die Speicherung von Teilen des elektronischen Angebots auf anderen Servern ist nur mit vorheriger schriftlicher Genehmigung des Konsortiums der Schweizer Hochschulbibliotheken möglich. Die Rechte für diese und andere Nutzungsarten der Inhalte liegen beim Herausgeber bzw. beim Verlag.

\section{SEALS}

Ein Dienst des Konsortiums der Schweizer Hochschulbibliotheken c/o ETH-Bibliothek, Rämistrasse 101, 8092 Zürich, Schweiz

retro@seals.ch

http://retro.seals.ch 


\title{
Positive-definite quadratic bundles over the plane
}

\author{
M.-A. Knus, M. Ojanguren and Raman Parimala
}

\section{Introduction}

Indecomposable, positive-definite quadratic spaces of ranks 3 and 4 over $\mathbb{R}[x, y]$ have been constructed in [5] and [13]. A natural question to ask is whether there exist indecomposable quadratic spaces of rank $>4$ over $\mathbb{R}[x, y]$ and whether the theorem of Krull-Schmidt holds for orthogonal decompositions of positive-definite quadratic spaces over $\mathbb{R}[x, y]$. (cf [9], p. 204.)

In $\$ 1$ of this paper we prove a Krull-Schmidt theorem for orthogonal sums of positive-definite quadratic spaces over $\mathbb{R}[x, y]$. In view of [8], Thm. 2.1 , it is enough to prove a similar theorem for positive-definite quadratic bundles over $\mathbb{P}_{\mathbb{R}}^{2}$. More generally, we prove that if $X$ is a projective scheme over $\mathbb{R}$ and $X_{\mathbb{C}}$ the complexification of $X$, then the theorem of Krull-Schmidt holds for positivedefinite $\sigma$-hermitian (resp. quadratic) bundles over $X_{\mathbb{C}}$ (resp. $X$ ). We also deduce that Witt-cancellation holds for positive-definite quadratic spaces over $\mathbb{R}[x, y]$. In $\S 2$, we exhibit a class of vector-bundles of rank 3 and 4 over $\mathbb{P}_{\mathbb{C}}^{2}$, associated to a pair of projective ideals of $\mathbb{H}[x, y]$, and show, using results of $\S 1$, that these bundles are stable. (The examples of rank 4 bundles over $\mathbb{P}_{\mathbb{C}}^{2}$ constructed here are interesting, particularly in view of the fact that in general it is not easy to decide the stability of bundles of rank $>3$.) In $\S 3$, we construct an example of a rank 6 , indecomposable quadratic space over $\mathbb{R}[x, y]$. The idea of the construction is to patch certain rank 3 and 4 quadratic spaces over $\mathbb{R}[x, y]$.

We are grateful to $R$. Sridharan for his contributions to this paper. We also thank W. Scharlau for explaining to us the content of [15].

\section{\$1. Krull-Schmidt theorem for positive-definite bundles over projective schemes}

Let $X$ be a projective scheme over $\mathbb{R}$ and let $X_{\mathbb{C}}$ denote the complexification Spec $\underset{\text { Spec } \mathbb{R}}{\mathbb{C}} \underset{X}{X}$ of $X$. Let $\sigma$ be the involution on $X_{\mathbb{C}}$ induced by the complex conjugation on $\mathbb{C}$ and $\pi$ the projection of $X_{\mathbb{C}}$ onto $X$. For any vector bundle $\mathscr{F}_{0}$ over $X$ we have a natural isomorphism $\rho: \pi^{*} \mathscr{F}_{0} \rightarrow \sigma^{*} \pi^{*} \mathscr{F}_{0}$, since $\pi \circ \sigma=\pi$. For 
any vector bundle $\mathscr{F}$ over $X_{\mathbb{C}}$ we denote by $\mathscr{F}^{\prime}$ the dual bundle and by $\mathscr{F}^{*}$ the pull-back $\sigma^{*} \mathscr{F}^{\prime}$ of $\mathscr{F}^{\prime}$ through $\sigma$. We define a natural isomorphism (cfr. [11]) $\tau:\left(\sigma^{*} \mathscr{F}\right)^{\prime} \rightarrow \mathscr{F}^{*}$ by

$$
\left(\sigma^{*} \mathscr{F}\right)^{\prime}=\mathscr{H}_{\sigma m}\left(\sigma^{*} \mathscr{F}, \pi^{*} \mathcal{O}_{\mathbf{X}_{\mathrm{c}}}\right) \stackrel{\mathscr{H}_{o m}\left(\sigma^{* \mathscr{F}}, \rho\right)}{\longrightarrow} \mathscr{H}_{\sigma m}\left(\sigma^{*} \mathscr{F}, \sigma^{*} \pi^{*} \mathcal{O}_{\mathbf{X}_{\mathrm{c}}}\right)=\sigma^{*} \mathscr{F}^{\prime}
$$

In [1.1] a $\sigma$-hermitian structure over $\mathscr{F}$ was defined as an isomorphism $\phi: \mathscr{F} \rightarrow$ $\sigma^{*} \mathscr{F}^{\prime}$ such that the diagram

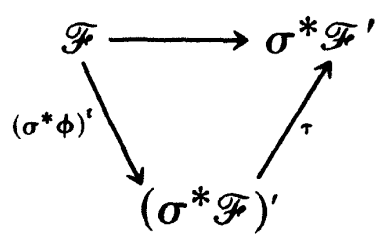

is commutative. It is convenient to give an equivalent definition, using the terminology of [15]. Let $\mathfrak{M}$ be the category of vector bundles over $X_{\mathbb{C}}$. Associating to every $\mathscr{F}$ the bundle $\mathscr{F}^{*}$ we get a functor $*: \mathfrak{M} \rightarrow \mathfrak{M}$. Let, for any $\mathscr{F}, \mathrm{i}_{\mathscr{F}}: \mathscr{F} \rightarrow \mathscr{F}^{* *}$ be the isomorphism defined by

$$
\mathscr{F}^{* *}=\sigma^{*}\left(\mathscr{F}^{*}\right)^{\prime} \underset{\tau_{* !}^{-1}}{\longrightarrow}\left(\sigma^{*} \mathscr{F}^{*}\right)^{\prime} \longrightarrow\left(\mathscr{F}^{\prime}\right)^{\prime} \longrightarrow \mathscr{F} .
$$

It is easily checked that $i$ is a natural transformation $i d \widetilde{\rightarrow}^{* *}$ satisfying $i_{*}^{*} i_{\mathscr{F}}=i d_{\mathscr{F}^{*}}$. Hence ${ }^{*}$ is a duality functor in the sense of [15]. We identify each bundle $\mathscr{F}$ with $\mathscr{F}^{* *}$ and each morphism $\phi$ of bundles with $\phi^{* *}$. For $\varepsilon= \pm 1$, we define an $\varepsilon$-hermitian structure on $\mathscr{F}$ as an isomorphism $\phi: \mathscr{F} \underset{\rightarrow}{\rightarrow} *$ such that $\phi^{*}=\varepsilon \phi$. A 1 -hermitian structure on $\mathscr{F}$ turns out to be the same as a $\sigma$-hermitian structure in the sense defined above and in [11] or [8]. If $x$ is a real closed point of $X_{\mathbb{C}}$, i.e. a closed point such that $\sigma(x)=x$, the fibre $\mathscr{F}_{x}$ at $x$ of a $\sigma$-hermitian bundle $\mathscr{F}$ carries a non-degenerate hermitian form. We say that $\mathscr{F}$ is positive definite if the fibre at every real closed point is positive definite. Since the signature of a hermitian form is locally constant, if $X_{\mathbb{R}}$ is connected, $\mathscr{F}$ is positive definite if and only if the induced form on the fibre of some real closed point of $X_{\mathbb{C}}$ is positive definite.

We assume, from now on, that $X$ has at least one real closed point.

For any bundle $\mathscr{F}$ we denote by $H(\mathscr{F})$ the hyperbolic bundle associated to $\mathscr{F}$. This is the bundle $\mathscr{F} \oplus \mathscr{F}^{*}$ with the hermitian structure defined by the matrix $\left(\begin{array}{ll}0 & 1 \\ 1 & 0\end{array}\right)$

LEMMA 1.1. Let $\mathcal{N}$ be an indecomposable vector bundle over $X_{\mathbb{C}}$ such that $\mathcal{N} \cong \mathcal{N}^{*}$. Then $\mathcal{N}$ carries a $\sigma$-hermitian structure. 
Proof. By Proposition 2.5 of [15], $\mathcal{N}$ carries a (1)- or a (-1)-hermitian form. If $\phi: \mathcal{N} \rightarrow \mathcal{N}^{*}$ is $(-1)$-hermitian, $i \phi$ is hermitian.

THEOREM 1.2. Let $(\mathscr{E}, \phi)$ be a positive-definite $\sigma$-hermitian bundle over $X_{\mathbb{C}}$. Then, there is a unique orthogonal decomposition

$$
(\mathscr{E}, \phi) \widetilde{\perp} \frac{1}{i}\left(\mathscr{C}_{i}, \phi_{i}\right)
$$

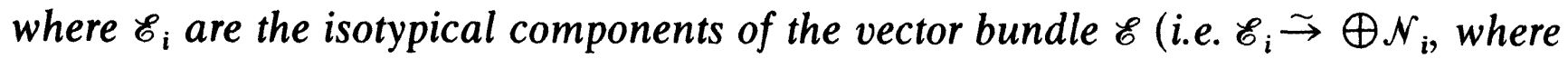
$\mathcal{N}_{i}$ are indecomposable and for $i \neq j, \mathcal{N}_{i} \not \supset \mathcal{N}_{j}$ ). Each $\mathscr{E}_{i}$ carries a positive-definite $\sigma$-hermitian structure which is unique up to isometry.

Proof. Since $X$ is a projective scheme, the category $\mathfrak{M}$ with the duality functor * defined above satisfies the assumptions (i)-(iii) of [15], page 272. Hence, by Theorem 3.2 of [15],

$$
(\mathscr{E}, \phi) \cong\left(\mathscr{C}_{1}, \phi_{1}\right) \perp \cdots \perp\left(\mathscr{C}_{n}, \phi_{n}\right),
$$

where each $\mathscr{C}_{i}$ is a direct sum of vector bundles isomorphic to a fixed indecomposable $\mathcal{N}_{i}$ or to its "dual" $\mathcal{N}_{i}^{*}$. By Theorem 3.3 of [15], if $\mathcal{N}_{i} \neq \mathcal{N}_{i}^{*}, \mathscr{C}_{i}$ contains a hyperbolic orthogonal summand. Since, by assumption, $\mathscr{E}$ is positive definite, this cannot happen and hence each $\mathscr{E}_{i}$ is isotypical. Since the orthogonal decomposition written above is unique, it suffices to prove the uniqueness for an isotypical vector bundle.

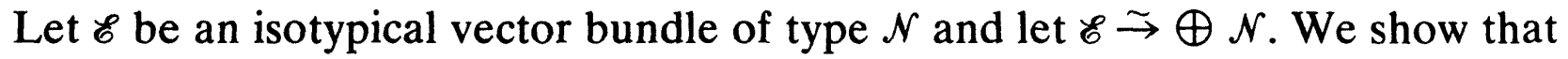
if $\mathscr{E}$ carries a positive-definite $\sigma$-hermitian structure, then it is unique. Since $\mathcal{N}$ is indecomposable, the ring $E=E n d \mathcal{N}$ is a local finite-dimensional $\mathbb{C}$-algebra. Let $\bar{E}=E / \mathrm{rad} E$. Then $\bar{E}$ is a finite-dimensional division algebra over $\mathbb{C}$ and hence $\bar{E} \leadsto \mathbb{C}$. One reduces the study of $\sigma$-hermitian structures on $\mathscr{E}$ to the study of hermitian-forms over a certain vector space $\bar{M}$ over $\bar{E}$ defined as follows (see $[15], 2.2,2.4)$. Let $\phi: \mathscr{E} \rightarrow \mathscr{C}^{*}$ be a $\sigma$-hermitian structure on $\mathscr{E}$. Then,

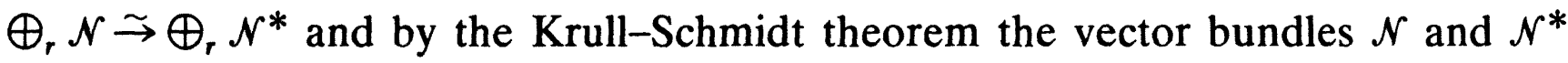
are isomorphic. Hence, by Lemma 1.1 , there exists an isomorphism $\phi_{0}: \mathcal{N} \neg \mathcal{N}^{*}$ which defines a $\sigma$-hermitian structure on $\mathcal{N}$. In what follows, we shall fix this $\sigma$-hermitian structure $\phi_{0}$ on $\mathcal{N}$. The isomorphism $\phi_{0}$ induces an involution $\tau$ on $E=$ End $\mathcal{N}$ defined as

$$
\tau f=f^{0}=\phi_{0}^{-1} \circ f^{*} \circ \phi_{0} .
$$

The map $f \rightarrow f^{0}$ satisfies $(f g)^{0}=g^{0} f^{0},\left(f^{0}\right)^{0}=f$ and for $\lambda \in \mathbb{C},(\lambda f)^{0}=\bar{\lambda} f^{0}, \bar{\lambda}$ denoting the complex conjugate of $\lambda$. This involution passes down to an involution on 
$\bar{E}=E / \operatorname{rad} E=\mathbb{C}$ which is just the complex conjugation on $\mathbb{C}$. Let $M=$ $\operatorname{Hom}(\mathcal{N}, \mathscr{E})$. Then $M$ is a right $E$-module and the isomorphism $\phi: \mathscr{E} \rightarrow \mathscr{C}^{*}$ induces an isomorphism $\phi_{1}: M \rightarrow \operatorname{Hom}_{E}(M, E)$ which is semilinear with respect to the involution $\tau$. The map $\phi_{1}$ is in fact defined as $\phi_{1}(f)(g)=\phi_{0}^{-1} \circ f^{*} \circ g$ for $f, g \in M$. It is easily verified that $\phi_{1}$ defines a hermitian form on the $E$-module $M$ with respect to the involution $\tau$ on $E$. Going modulo the radical of $E$, we obtain on $\bar{M}=M /(\operatorname{rad} E) M$ a hermitian form over $\mathbb{C}$.

Two $\sigma$-hermitian structures on $\mathscr{E}$ are isometric if and only if the corresponding hermitian forms on $\bar{M}$ are isometric ([15], 2.2). If the form on $\mathscr{E}$ is positivedefinite, then the form on $\bar{M}$ is either positive or negative-definite. In fact, if $\bar{M}$ represents zero, then $\bar{M}$ contains a hyperbolic summand and so does $\mathscr{E}$ by [15], Prop. 2.4. If $\phi$ and $\phi^{\prime}$ are two positive definite forms on $\mathscr{E}$, the corresponding forms on $\bar{M}$ are either both positive-definite or both negative-definite: otherwise the form corresponding to $\phi \perp \phi^{\prime}$ on $\mathscr{E} \perp \mathscr{E}$ would be isotropic. Since, up to isometry, there is a unique positive or negative-definite hermitian form on $\bar{M}$, it follows that there is a unique positive definite $\sigma$-hermitian structure over $\mathscr{C}$. This proves Theorem 1.2.

COROLLARY 1.3. A vector bundle over $X_{\mathbb{C}}$ carries at the most one positivedefinite $\sigma$-hermitian structure.

COROLLARY 1.4 (Krull-Schmidt theorem). Any $\sigma$-hermitian positivedefinite bundle $(\mathscr{E}, \phi)$ over $X_{\mathbb{C}}$ has a decomposition

$$
(\mathscr{E}, \phi)=\perp\left(\mathcal{N}_{i}, \nu_{i}\right)
$$

into indecomposable $\sigma$-hermitian bundles. The summands $\left(\mathcal{N}_{i}, \nu_{i}\right)$ are unique up to isometries and permutations.

COROLLARY 1.5. The Krull-Schmidt theorem holds for positive-definite $\sigma$-hermitian spaces over $\mathbb{C}[x, y]$.

Proof. By (3.1) of [8] any positive-definite $\sigma$-hermitian space over $\mathbb{C}[x, y]$ has, up to isometry, a unique extension to $\mathbb{P}_{\mathbb{C}}^{2}$. Hence the assertion follows from 1.4.

The following theorem and corollaries give the corresponding results for positive-definite quadratic bundles.

THEOREM 1.6. Let $(\mathscr{E}, \phi)$ be a positive-definite quadratic bundle over $X$. Then, there is a unique orthogonal decomposition

$$
(\mathscr{E}, \phi)=\frac{1}{i}\left(\mathscr{E}_{i}, \phi_{i}\right)
$$


where $\mathscr{E}_{i}$ are the isotypical components of the vector bundle $\mathscr{E}$. The components $\mathscr{E}_{i}$ carry a positive-definite quadratic structure, unique up to isometry.

A proof on the same lines as of Theorem 1.2 can be given. Let now $\mathfrak{M}$ be the category of real vector bundles over $X$ and, for any such bundle $\mathscr{E}$ let $\mathscr{E}^{*}=\mathscr{E}^{\prime}=$ $\mathscr{H}_{0 m}\left(\mathscr{E}, \mathcal{O}_{\mathbf{X}}\right)$ be the dual of $\mathscr{E}$. By Theorem 3.2 of [15] one reduces immediately to

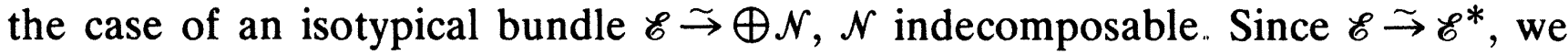
have $\mathcal{N} \widetilde{\rightarrow} \mathcal{N}^{*}$ and since End $\mathcal{N}$ is local, $\mathcal{N}$ carries either a quadratic or a symplectic structure $\phi_{0}: \mathcal{N} \leadsto \mathcal{N}^{*}$. Then $\phi_{0}$ gives rise to an involution $\tau$ of $E=\operatorname{End} \mathcal{N}$, which passes down to an involution of $\bar{E}=E / \mathrm{rad} E$. It is clear that $\bar{E} \neg \mathbb{R}, \mathbb{C}$, or $\mathbb{H}$. If $\bar{E}=\mathbb{R}$, the involution is trivial. If $\bar{E}=\mathbb{C}$, the involution must be complex conjugation. And if $\bar{E} \neg \mathbb{H}$, the involution on $\mathbb{H}$ is either trivial or is a conjugate of the canonical involution. The isometry classes of quadratic structures on $\mathscr{E}$ correspond to isometry classes of positive-definite or negative-definite forms on $\bar{M}=M /(\operatorname{rad} E) M$, where $M=\operatorname{Hom}(\mathcal{N}, \mathscr{E})$. The existence of orthogonal bases for hermitian forms shows that there is unique positive- or negative-definite $\tau$-hermitian form on $\bar{M}$. It follows that there is a unique positive-definite quadratic structure over $\mathscr{E}$.

COROLLARY 1.7. A vector bundle over $X$ carries at the most one positivedefinite quadratic structure.

COROLLARY 1.8. The Krull-Schmidt theorem holds for positive-definite quadratic bundles over $X$.

COROLLARY 1.9. The Krull-Schmidt theorem holds for positive-definite quadratic spaces over $\mathbb{R}[x, y]$.

\section{§2. Some stable bundles of rank 3 and 4 associated to projective ideals of $\mathbb{H}[x, y]$}

We recall that a bundle $\mathscr{E}$ over $\mathbb{P}_{\mathbb{C}}^{r}$ is said to be stable if, for every coherent subsheaf $\mathscr{F} \neq 0$ of $\mathscr{E}$ such that $\mathscr{E} / \mathscr{F}$ is torsionfree we have $c_{1}(\mathscr{F}) /$ rank $\mathscr{F}<$ $c_{1}(\mathscr{E}) /$ rank $\mathscr{E}$. In [8] to each non-free projective ideal $P$ of $\mathbb{H}[[x, y]$ was associated a rank 2 stable bundle $\mathscr{C}(P)$ with a positive-definite $\sigma$-hermitian structure. We recall the construction of these bundles, which in [8] were called $\mathfrak{\beta}$-bundles. Let $\phi: \mathbb{C} \otimes \mathbb{H} \rightarrow M_{2}(\mathbb{C})$ be the isomorphism given by

$$
\phi(s \otimes(u+v j))=s\left(\begin{array}{rr}
u & v \\
-\bar{v} & \bar{u}
\end{array}\right) u, v \in \mathbb{C}
$$


Let $H=\mathbb{H}[x, y]$ and $C=\mathbb{C}[x, y]$. For any projective ideal $P$ of $H, C \otimes P$ is an $M_{2}(C)$-module via $\phi$. Hence, there is a $\phi$-semilinear isomorphism $\Psi_{P}: C \otimes$ $P \neg M_{2}(C)$. We shall call such a map a splitting of $P$. By Galois cohomology, we associate to the splitting $\Psi_{P}$ the cocycle

$$
\alpha_{P}=\sigma \Psi_{P}(\sigma \otimes 1) \Psi_{P}^{-1}(1) \in G L_{2}(C)
$$

where $\sigma$ is the complex conjugation on $\mathbb{C}$ and the transported action $\phi(\sigma \otimes 1) \phi^{-1}$ on $\boldsymbol{M}_{2}(C)$. The map $\Psi_{\mathrm{P}}$ can be chosen such that $\alpha_{P}$ is positive-definite hermitian of determinant one. Such a splitting is called a normalized splitting. Hence, $\alpha_{P}$ defines a $\sigma$-hermitian structure on $\mathbb{A}_{\mathbb{C}}^{2}$. This structure can be uniquely extended to $\mathbb{P}_{\mathbb{C}}^{2}([8])$ and the extension is the complex bundle $\mathscr{C}(P)$. Notice that by $(1.2) \mathscr{E}(P)$ carries a unique positive-definite $\sigma$-hermitian structure. Let now $P$ and $Q$ be two projective ideals in $H$. The reduced norm $\mathrm{Nr}$ introduced in [6] defines a quadratic form on the $\mathbb{R}[x, y]$-module of rank $4 \operatorname{Hom}_{H}(P, Q)$. If $\Psi_{P}: \mathbb{C} \otimes P \simeq M_{2}(C)$ and $\Psi_{Q}: \mathbb{C} \otimes Q \stackrel{\sim}{\rightarrow} M_{2}(C)$ are normalized splittings of $P$ and $Q$, then, for any $f \in$ $\operatorname{Hom}_{H}(P, Q), \operatorname{Nr}(f)=\operatorname{det} \Psi_{Q}(1 \otimes f) \Psi_{P}^{-1}(1)$. This quadratic space is indecomposable if $P$ and $Q$ are non-free and not isomorphic. If $P \simeq Q$ and $P$ is non-free, then this space decomposes as $\langle 1\rangle \perp \bar{q}$, where $\bar{q}$ is the orthogonal complement of the submodule $\mathbb{R}[x, y]$ of $\operatorname{End}_{H}(P)$ for the reduced norm on the algebra $\operatorname{End}_{H}(P)$. It is shown in [6] that $\bar{q}$ is indecomposable. These indecomposable quadratic spaces of ranks 3 and 4 extend uniquely to indecomposable quadratic bundles over $\mathbb{P}_{\mathbb{R}}^{2}$, denoted respectively by $\mathscr{F}(P, Q)$ and $\mathscr{F}(P)$. Let $\pi: \mathbb{P}_{\mathbb{C}}^{2} \rightarrow \mathbb{P}_{\mathbb{R}}^{2}$ be the projection and let $\pi^{*} \mathscr{F}(P, Q)=\mathscr{G}(P, Q)$ and $\pi^{*} \mathscr{F}(P)=\mathscr{G}(P)$. We shall show that these bundles are stable.

THEOREM 2.1. The bundle $\mathscr{G}(P, Q)$ is isomorphic to $\mathscr{E}(P) \otimes \mathscr{E}(Q)$.

COROLLARY 2.2. We have $c_{2}\left(\mathscr{G}(P, Q)=2\left(c_{2}\left(\mathscr{E}(P)+c_{2}(\mathscr{E}(Q))\right.\right.\right.$ and $c_{2}(\mathscr{G}(P))=4 c_{2}(\mathscr{E}(P))$.

Proof. For 2-bundles $\mathscr{E}$ and $\mathscr{F}$ on $\mathbb{P}_{\mathbb{C}}^{2}$, if $c_{1}(\mathscr{E})=c_{1}(\mathscr{F})=0$, then $c_{2}(\mathscr{E} \otimes \mathscr{F})$ is given by $2\left(c_{2}(\mathscr{E})+c_{2}(\mathscr{F})\right)$.

Theorem (2.1) is a consequence of the following results. The first one is implicitly contained in [7], (1.12).

LEMMA 2.3. Let $P$ be a projective ideal of $H, \Psi_{P}$ a normalized splitting of $P$ and $\alpha_{P} \in G L_{2}(C)$ the corresponding cocycle. Then there is a basis $e_{1}, e_{2}$ of $P$ as a 
$C$-module such that the matrix of the $\sigma$-hermitian form $a_{P}$ on $P$ defined by

$$
\begin{aligned}
& a_{P}\left(e_{i}, e_{i}\right)=\left(\Psi_{P}\left(e_{i}\right), \Psi_{P}\left(e_{i}\right)\right) \quad i=1,2 \\
& a_{P}\left(e_{1}, e_{2}\right)=\left(\Psi_{P}\left(e_{1}\right), \Psi_{P}\left(e_{2}\right)\right)-i\left(\Psi_{P}\left(e_{1}\right), \Psi_{P}\left(i e_{2}\right)\right)
\end{aligned}
$$

where, for $u, v \in M_{2}(C),(u, v)=\frac{1}{2}(\operatorname{det}(u+v)-\operatorname{det} u-\operatorname{det} v)$, is $\alpha_{P}$.

Let $\alpha_{P}=\alpha+i \beta$ with $\alpha, \beta \in M_{2}(\mathbb{R}[x, y])$. Then the symmetric matrix $\left(\begin{array}{rr}\alpha & \beta \\ -\beta & \alpha\end{array}\right)$ represents the reduced norm on $P$ with respect to the basis $e_{1}, e_{2}, e_{3}=i e_{1}, e_{4}=i e_{2}$ of $P$ over $\mathbb{R}[x, y]$.

The next lemma is an immediate consequence of (2.3) and of the definition of the reduced norm on $\operatorname{Hom}_{H}(P, Q)$ by means of the splittings $\Psi_{P}$ and $\Psi_{Q}$.

LEMMA 2.4. Let $f \in \operatorname{Hom}_{H}(P, Q)$ and $a_{P}, a_{Q}$ the hermitian forms given in (2.1). Then, for any $u, v \in P$

$$
a_{Q}(f(u), f(v))=\operatorname{Nr}(f) a_{P}(u, v) .
$$

The module $P^{\prime}=\operatorname{Hom}_{C}(P, C)$ is a projective right $H$-module (with the action $(f \lambda)(x)=f(\lambda x), \lambda \in H)$. We now compute its cocycle.

LEMMA 2.5. Let $\Psi_{P}$ be a splitting of $P$ with cocycle $\alpha_{P}$. Then, there is a splitting $\Psi_{P^{\prime}}$ of $P^{\prime}$ with cocycle $\alpha_{P^{\prime}}=\alpha_{P}^{-1}$.

Proof. Let $T: M_{2}(C) \stackrel{\rightarrow}{\rightarrow} \operatorname{Hom}_{C}\left(M_{2}(C), C\right)$ be the isomorphism given by the trace, i.e. $T_{a}(b)=\operatorname{Tr}(a b), a, b \in M_{2}(C)$. Let $P^{\wedge}=\operatorname{Hom}_{\mathbb{R}[x, y]}(P, \mathbb{R}[x, y])$. Then the map $\Psi_{P^{\wedge}}=T^{-1}\left(\Psi_{P}\right)^{\wedge}$ (where ${ }^{\wedge}$ means dualization with respect to $\mathbb{R}[x, y]$ ) is a splitting of $\boldsymbol{P}^{\wedge}$ and one computes that the corresponding cocycle is $\alpha_{P}^{-1}$. Let now $t: P^{\prime} \stackrel{\rightarrow}{\rightarrow} P^{\wedge}$ be the isomorphism (of $H$-modules) induced by the trace $\mathbb{C} \rightarrow \mathbb{R}$. Then the map $\Psi_{P^{\prime}}=\Psi_{P^{\wedge}} \circ(1 \otimes t)$ is a splitting of $P^{\prime}$ such that $\alpha_{P^{\prime}}=\alpha_{P^{\wedge}}=\alpha_{P^{-1}}^{-1}$.

Let now $a_{P^{\prime}}$ be the hermitian structure on $P^{\prime}$ given by

$$
a_{P^{\prime}}\left(e_{i}^{\prime}, e_{j}^{\prime}\right)=\frac{1}{2}\left(\alpha_{P^{\prime}}\right)_{j, i}=\frac{1}{2}\left(\alpha_{P}^{-1}\right)_{j, i}
$$

where $e_{i}^{\prime}, i=1,2$ is the dual basis of the basis $e_{i}, i=1,2$ given in (2.3). Let $S$ be the $\sigma$-hermitian space obtained by extending the reduced norm $\mathrm{Nr}$ on $\operatorname{Hom}_{H}(P, Q)$ to $\mathbb{C} \bigotimes_{\mathbb{R}} \operatorname{Hom}_{H}(P, Q)$, i.e. $S(\lambda \otimes f)=\lambda \bar{\lambda} \operatorname{Nr}(f)$. 
LEMMA 2.6. The map $\rho: \mathbb{C} \otimes \operatorname{Hom}_{H}(P, Q) \stackrel{\sim}{\rightarrow} \operatorname{Hom}_{C}(P, Q) \stackrel{\sim}{\rightarrow} P^{\prime} \otimes_{C} Q$ where the first map is the multiplication and the second is the canonical map, is an isomorphism of $\sigma$-hermitian spaces $\rho: S \stackrel{\sim}{\rightarrow} a_{P^{\prime}} \otimes a_{Q}$.

Proof. For any basis $\left\{e_{i}\right\}$ of $P, \rho$ is given by $\rho(\lambda \otimes f)=\sum_{i} e_{i}^{*} \otimes f\left(\lambda e_{i}\right)$. Choosing the basis given in (2.3), we have, using (2.4),

$$
\begin{aligned}
\left(a_{P^{\prime}} \otimes a_{Q}\right)\left(\sum_{i} e_{i}^{\prime} \otimes f\left(\lambda e_{i}\right)\right) & =\sum_{i, j} a_{P^{\prime}}\left(e_{i}^{\prime}, e_{j}^{\prime}\right) a_{Q}\left(f\left(\lambda e_{i}\right), f\left(\lambda e_{j}\right)\right) \\
& =\operatorname{Nr}(f) \lambda \bar{\lambda} \sum_{i, j} a_{P^{\prime}}\left(e_{i}^{\prime}, e_{j}^{\prime}\right) a_{Q}\left(e_{i}, e_{j}\right)=\operatorname{Nr}(f) \lambda \bar{\lambda} .
\end{aligned}
$$

This shows that $\rho$ is an isometry.

Theorem (2.1) now follows from (2.6) noting that the extension of a positive definite $\sigma$-hermitian form from $\mathbb{A}_{\mathbb{C}}^{2}$ to $\mathbb{P}_{\mathbb{C}}^{2}$ is unique and that $\mathscr{C}\left(P^{*}\right) \cong \mathscr{C}(P)$.

To show that the bundles $\mathscr{G}(P, Q)$ and $\mathscr{G}(P)$ are stable, we begin with

LEMMA 2.7. Let $K$ be a field of characteristic $\neq 2$ and let $(\mathscr{E}, \phi)$ be a quadratic bundle of rank 2 over $\mathbb{P}_{K}^{r}$. If $(\mathscr{E}, \phi)$ is anisotropic, $(\mathscr{E}, \phi)$ is extended from $K$. If

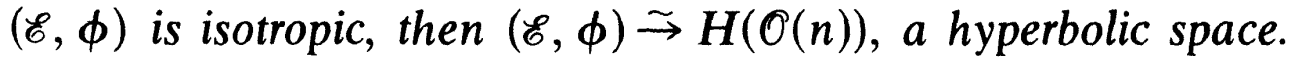

Proof. The first part of the lemma is proved in ([8],2.4). If $(\mathscr{E}, \phi)$ is isotropic, then restricted to each affine piece $D\left(x_{i}\right)$, the quadratic form can be given by the matrix $\left(\begin{array}{ll}0 & 1 \\ 1 & 0\end{array}\right)$. One then easily checks that $(\mathscr{E}, \phi) \Im H(\mathscr{O}(n))$ for some $n$.

LEMMA 2.8. Let $K$ be a field of characteristic $\neq 2$ and let $\mathscr{E}$ be an indecomposable anisotropic quadratic bundle over $\mathbb{P}_{K}^{r}$. Then $\mathscr{C}$ has no non-zero section.

Proof. Evaluating the quadratic form on a global section one gets a global function on $\mathbb{P}_{K}^{r}$, hence a constant. This constant must be zero, since the bundle is indecomposable as a quadratic bundle. The section has to be zero since the form is anisotropic.

For any bundle $\mathscr{E}$ over $\mathbb{P}_{\mathbb{C}}^{2}$ the "type" of $\mathscr{E}$ is the pair of Chern classes $\left(c_{1}(\mathscr{E})\right.$, $\left.c_{2}(\mathscr{E})\right)$.

THEOREM 2.9. The bundles $\mathscr{G}(P)$ are stable rank 3 bundles of type $(0,8 n)$, where $c_{2}(\mathscr{E}(P))=2 n, \mathscr{E}(P)$ denoting the $\mathfrak{B}$-bundle associated to a non-free projective ideal $P$ of $\mathbb{H}[x, y]$. The bundles $\mathscr{C}(P, Q)$ are stable rank 4 of type $(0,4(m+n))$ if $P$ and $Q$ are non-isomorphic, non-free, $\mathscr{E}(P)$ of type $(0,2 n)$ and $\mathscr{C}(Q)$ of type $(0,2 m)$. 
Proof. Since $\mathscr{C}(P)$ supports a quadratic form it follows that $c_{1}(\mathscr{E}(P))=0$. If we consider global sections, we have $H^{0}\left(\mathbb{P}_{\mathbb{C}}^{2}, \mathscr{G}(P)\right) \stackrel{\sim}{\rightarrow} \otimes H^{0}\left(P_{\mathbb{R}}^{2}, \mathscr{F}(P)\right)=0$ by Lemma 2.7, since $\mathscr{F}(P)$ supports an anisotropic indecomposable quadratic form. Further, being a quadratic bundle, $\mathscr{G}(P) \sim \mathscr{G}(P)^{\prime}$. Hence $\mathscr{G}(P)$ is stable by [12], 1.2.6.

We shall now show that $\mathscr{G}(P, Q)$ is stable for $P, Q$ non-isomorphic, non-free. We show that for every subsheaf $\mathscr{F}$ of $\mathscr{G}=\mathscr{G}(P, Q)$ with the quotient $\mathscr{G}(P, Q) / \mathscr{F}$ torsion free, $c_{1}(\mathscr{F}) /$ rank $\mathscr{F}<c_{1}(\mathscr{G}) /$ rank $\mathscr{G}$. Since $\mathbb{P}_{\mathbb{C}}^{2}$ is regular of dimension 2 , such a sheaf is locally free. Hence it suffices to show that for any locally free subsheaf $\mathscr{F}$ of $\mathscr{G}, c_{1}(\mathscr{F})<0$. If $\mathscr{F}$ is a line bundle with $c_{1}(\mathscr{F})=n$, necessarily $n<0$ since, otherwise, $\mathscr{F}$ and hence $\mathscr{G}$ would have a non-zero global section. If $\mathscr{F}$ is of rank 3 we have a surjection $\mathscr{G}^{\prime} \rightarrow \mathscr{F}^{\prime} \rightarrow 0$ whose kernel is a line bundle $\mathscr{L}$. Since $\mathscr{G}^{\prime} \sim \mathscr{G}$ also does not admit of global sections, it follows that $c_{1}(\mathscr{L})<0$. Hence $c_{1}\left(\mathscr{F}^{\prime}\right)>0$ so that $c_{1}(\mathscr{F})=-c_{1}\left(\mathscr{F}^{\prime}\right)<0$. Let $\mathscr{F}$ be of rank 2 . The bundle $\mathscr{G}$ restricted to a real line $L$ of $\mathbb{P}_{\mathbb{C}}^{2}$ is trivial, since $\mathscr{G}$ supports an anisotropic quadratic form ([16], Prop. 5). The restriction of $\mathscr{F}$ to $L$ is isomorphic to $\mathscr{O}(n) \oplus \mathscr{O}(m)$. Since $\left.\mathscr{F}\right|_{L}$ is a subsheaf of $\left.\left.\mathscr{G}\right|_{L} \cong \oplus \mathcal{O}\right|_{L}$, we have $c_{1}(\mathscr{F})=n+m \leqslant 0$. Suppose that $c_{1}(\mathscr{F})=0$. Then $\mathscr{F}$ is a rank 2 bundle with no global sections and with $c_{1}(\mathscr{F})=0$. Hence $\mathscr{F}$ is a stable bundle $([12], 1.2 .5)$. The quadratic structure on $\mathscr{F}(P, Q)$ extends to a positive-definite $\sigma$-hermitian structure, denoted by $\phi$, on $\mathscr{G}(P, Q)$. The restriction of $\phi$ to $\mathscr{F}$ induces a map $\mathscr{F} \rightarrow \sigma^{*} \mathscr{F}^{*}=\mathscr{F}^{*}$. This map cannot be zero since $\mathscr{F}$ is anisotropic (positive-definite). By the corollary to Lemma 1.2.8 of [12], $\phi$ is an isomorphism and $(\mathscr{F}, \phi \mid \mathscr{F})$ splits off as an orthogonal summand of $(\mathscr{G}, \phi)$. Then, $\mathscr{G} \breve{\rightarrow} \mathscr{F} \perp \mathscr{F}_{1}$. The bundle $\mathscr{G}$ supports a quadratic form, namely the extension of the quadratic structure on $\mathscr{F}(P, Q)$. The bundle $\mathscr{F}$ cannot support a quadratic structure, since, otherwise, $\mathscr{F} \stackrel{\rightarrow}{\rightarrow} H(O(n))$ by Lemma 2.7 contradicting the stability of $\mathscr{F}$. Thus, by the uniqueness of the quadratic structure

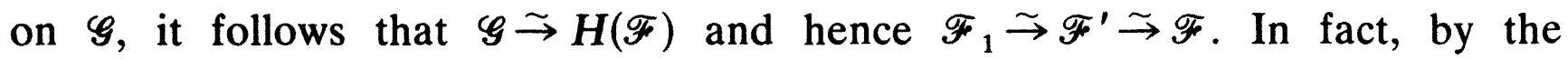
uniqueness of the positive-definite structure (see (1.6)) $\left(\mathscr{F}_{1}, \phi \mid \mathscr{F}_{1}\right) \stackrel{\sim}{\rightarrow}(\mathscr{F}, \phi \mid \mathscr{F})$

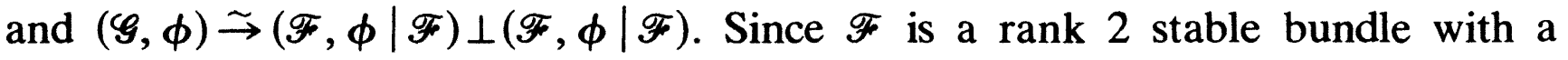
positive-definite $\sigma$-hermitian structure, it follows by [8] that $\mathscr{F}$ is a $\mathfrak{B}$-bundle, i.e. $\mathscr{F} \neg \mathscr{C}\left(P_{0}\right)$, where $P_{0}$ is some non-free projective ideal of $\mathbb{H}[x, y]$. By [8],

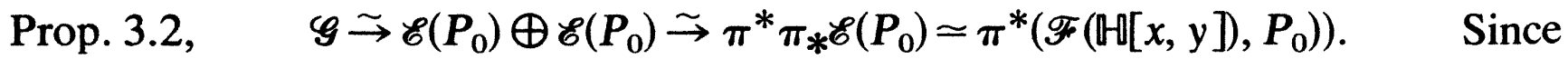
End $\left(\mathscr{C}\left(P_{0}\right) \oplus \mathscr{E}\left(P_{0}\right)\right) \stackrel{\sim}{\rightarrow} M_{2}(\mathbb{C})$, the isomorphism classes of vector-bundles on $\mathbb{P}_{\mathbb{R}}^{2}$ with $\pi^{*}(\mathscr{E}) \stackrel{\sim}{\rightarrow} \mathscr{E}\left(P_{0}\right) \oplus \mathscr{E}\left(P_{0}\right)$ are classified by $H^{1}\left(\mathbb{Z} / 2 \mathbb{Z}, G L_{2}(\mathbb{C})\right.$ for an action on $G L_{2}(\mathbb{C})$ which is the restriction of an action on $M_{2}(\mathbb{C})$. Since $\pi^{*}(\mathscr{E}) \stackrel{\sim}{\rightarrow} \mathscr{C}\left(P_{0}\right) \oplus$ $\mathscr{E}\left(P_{0}\right)$ is $\mathbb{C}$-linear, $\mathbb{Z} / 2 \mathbb{Z}$ acts on $\mathbb{C} \subset M_{2}(\mathbb{C})=$ End $\left(\mathscr{C}\left(P_{0}\right)+\mathscr{E}\left(P_{0}\right)\right)$ by conjugation, and hence the action on $M_{2}(\mathbb{C})$ is of the form $\alpha \rightarrow u \bar{\alpha} u^{-1}$ for some fixed $u \in G L_{2}(\mathbb{C})$. It is easily checked that in this case $H^{1}\left(\mathbb{Z} / 2 \mathbb{Z}, G L_{2}(\mathbb{C})\right)=0$. Hence,

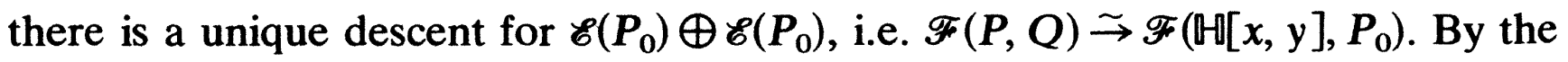


uniqueness of the positive-definite quadratic structure on a vector-bundle over $\mathbb{P}_{\mathbb{R}}^{2}$ $[(1.7)]$, it follows that $\mathscr{F}(P, Q)$ is isomorphic as a quadratic bundle to $\mathscr{F}\left(\mathbb{R}[x, y], P_{0}\right)$. By restricting these bundles to $\mathbb{A}_{\mathbb{R}}^{2}$ and using $([6]$, Thm. 4.6), it follows that $P$ or $Q$ is free, a contradiction. The statement in the theorem regarding the second Chern classes of $\mathscr{G}(P)$ and $\mathscr{G}(P, Q)$ was proved in (2.2).

\section{§3. An example of an indecomposable quadratic space of rank 6 over $\mathbb{R}[x, y]$}

LEMMA 3.1. Let $R$ be a local domain in which 2 is invertible and let $q_{1}, q_{2}$ be quadratic spaces over $R[x]$ such that $q_{1} \perp q_{2}$ is anisotropic. If $q_{1}(v)+q_{2}(w)$ is a unit of $R[x]$, then $q_{1}(v)$ or $q_{2}(w)$ is a unit of $R[x]$.

Proof. Let $K$ denote the quotient field of $R$. Since $R$ is local, if bar denotes

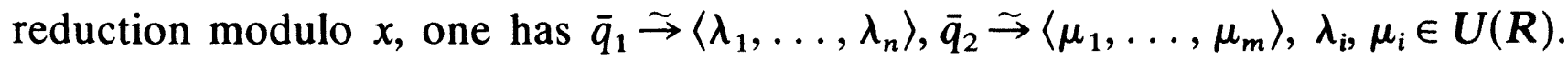
By a theorem of Harder, we have, over $K[x], \quad q_{1} \widetilde{\rightarrow}\left\langle\lambda_{1}, \ldots, \lambda_{n}\right\rangle$, $q_{2} \stackrel{\sim}{\rightarrow}\left\langle\mu_{1}, \ldots, \mu_{m}\right\rangle$. Thus, there exist $\theta_{i}, \phi_{i} \in K[x]$ such that $q_{1}(v)=\sum \lambda_{i} \theta_{i}^{2}$ and $q_{2}(w)=\sum \mu_{i} \phi_{i}^{2}$. Since the forms $q_{1}$ and $q_{2}$ are anisotropic over $K[x]$, if $q_{1}(v)=$ $a_{0}+a_{1} x+\cdots+a_{r} x^{r}$, then $q_{2}(w)=b_{0}-a_{1} x-\cdots-a_{r} x^{r}$, and $a_{r}=\sum \lambda_{i} c_{i}^{2}=-\sum \lambda_{i} d_{i}^{2}$, where $c_{i}, d_{i}$ denote the leading coefficients of $\theta_{i}$ and $\phi_{i}$ respectively. Then, $\bar{q}_{1} \perp \bar{q}_{2}$ represents zero over $K$ and hence $q_{1} \perp q_{2}$ represents zero over $K[x]$, contradicting the assumption that $q_{1} \perp q_{2}$ is anisotropic.

The next lemma is a generalization of Proposition 1.1 of [13].

LEMMA 3.2. Let $A$ be a normal ring in which 2 is invertible. Every quadratic space of rank 2 over $A\left[X_{1}, \ldots, X_{n}\right]$ is extended from $A$.

Proof. By [3,4.15, Remark 4] we may assume that $A$ is local. Let $K$ be the field of fractions of $A$ and $M$ a quadratic space of rank 2 over $A[X], X$ denoting $\left(X_{1}, \ldots, X_{n}\right)$. If the signed discriminant of $M_{K}$ is trivial, by [2, Proposition 5.1] $M$ is of the form $H(I)$, where $I$ is a projective ideal of $A[X]$. Since Pic $A=$ Pic $A[X], M$ is extended. If the signed discriminant $d$ of $M_{K}$ is not a square in $K$, put $L=K[\sqrt{ } d]$ and $B=A[\sqrt{ } d]$. Then $B$ is the integral closure of $A$ in $L$ hence is a normal semilocal ring. The signed discriminant of $M_{B}$ is trivial and hence $M_{B}$ is of the form $H(I)$, where $I$ is a projective ideal of $B[X]$. Since Pic $B[X]=$ Pic $B=$ $0, M_{B}=H(B[X])$. This shows that $M$ is represented by an element of $H^{1}\left(\mathrm{Gal}(L / K), \mathrm{O}_{2}(B[X])\right)$. But $\mathrm{O}_{2}(B[X])=\mathrm{O}_{2}(B)$ (compare [11], §1) and hence $M$ is in the image of $H^{1}\left(\mathrm{Gal}(L / K), O_{2}(B)\right)$ in $H^{1}\left(\mathrm{Gal}(L / K), O_{2}(B[X])\right)$. This shows that $M$ is extended from $A$. 
Given a pair $f, g$ of polynomials in $\mathbb{R}[x, y]$, let $\alpha_{f, g}$ (respectively $\beta_{f, g}$ ) denote the rank 3 (rank 4) quadratic spaces over $\mathbb{R}[x, y]$ defined as the orthogonal complement of the identity in End $\left(P_{f, g}\right)$ (respectively reduced norm on $\left.P_{f, g}\right)$, where $P_{f, g}$ is the projective ideal of $\mathbb{H}[x, y]$ defined as the kernel of the $\mathbb{H}[x, y]$ linear map $\mathbb{H}[x, y]^{2} \rightarrow \mathbb{H}[x, y]$ given by $(1,0) \rightarrow f+i,(0,1) \rightarrow g+j([8], 1.2)$. Then $\alpha=\alpha_{x, y}$ is an indecomposable quadratic space over $\mathbb{R}[x, y]$. This space remains indecomposable over $\mathbb{R}[x]_{\left(1+x^{2}\right)}[y]$. In fact, if it decomposes as $\alpha^{\prime} \perp \alpha^{\prime \prime}$, then the ranks of $\alpha^{\prime}$ and $\alpha^{\prime \prime}$ are 1 or 2 and hence, by Lemma 3.2, $\alpha$ is extended from $\mathbb{R}[x]_{\left(1+x^{2}\right)}$. Since over $\mathbb{R}\left[x, 1 / 1+x^{2}\right][y], P_{x, y}$ is free $([7], \S 5), \alpha$ is $\cong\langle 1,1,1\rangle$ over this ring. Therefore by $[3,4.15$, Remark 4$], \alpha$ is extended from $\mathbb{R}$, contrary to the assumption. The form $\beta=\beta_{x \sqrt{ } 2, y}$ is an indecomposable quadratic space over $\mathbb{R}[x, y]$ which is isometric to $\langle 1,1,1,1\rangle$ over $\mathbb{R}\left[x, 1 / 2+x^{2}\right][y]$. We claim that $\beta$ remains indecomposable over $\mathbb{R}[x]_{\left(2+x^{2}\right)}[y]$. Suppose that $\beta=\beta^{\prime} \perp \beta^{\prime \prime}$ over $\mathbb{R}[x]_{\left(2+x^{2}\right)}[y]$. If rank $\beta^{\prime}=\operatorname{rank} \beta^{\prime \prime}=2$ the same argument as above shows that $\beta$ is extended from $\mathbb{R}$, which is absurd. If rank $\beta^{\prime}=1$, then $\beta$ represents a unit over $\mathbb{R}[x]_{\left(2+x^{2}\right)}[y]$ and therefore, by [6], (3.19) $P_{x \sqrt{ } 2, y}$ is free over $\mathbb{H}[x]_{\left(2+x^{2}\right)}[y]$ and, in particular, extended from $\mathbb{H}$. Since it is also free over $\mathbb{H}\left[x, 1 / 2+x^{2}\right][y]([7], \S 5)$, by Quillen's theorem $P_{x \sqrt{ } 2, y}=\mathbb{H}[x, y]$, contrary to the assumption.

We define a quadratic space over $\mathbb{R}[x, y]$ of rank 6 as follows: we consider the covering

$$
\operatorname{Spec} \mathbb{R}[x, y]=\operatorname{Spec} \mathbb{R}[x, y]\left[1 / 1+x^{2}\right] \cup \operatorname{Spec} \mathbb{R}[x, y]\left[1 / 2+x^{2}\right] .
$$

We take the space $\beta \perp 1 \perp 1$ over Spec $\mathbb{R}[x, y]\left[1 / 1+x^{2}\right]$ and the space $\alpha \perp \alpha$ over

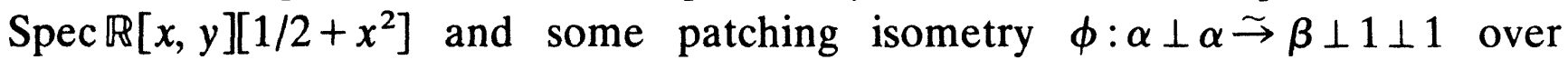
$\operatorname{Spec} \mathbb{R}[x, y]\left[1 /\left(1+x^{2}\right)\left(2+x^{2}\right)\right]$ (note that both quadratic spaces are equivalent to the identity over this intersection) to get a quadratic space $\gamma$ of rank 6 over $\operatorname{Spec} \mathbb{R}[x, y]$.

We show that $\gamma$ is indecomposable. Suppose that $\gamma$ represents a unit of $\mathbb{R}[x, y]$. Since $\gamma \stackrel{\sim}{\rightarrow} \alpha \perp \alpha$ over $\mathbb{R}[x]_{\left(1+x^{2}\right)}[y]$, it follows that $\alpha \perp \alpha$ represents a unit of $\mathbb{R}[x]_{\left(1+x^{2}\right)}[y]$ and since $\alpha \perp \alpha$ is anisotropic, by Lemma 3.1, $\alpha$ represents a unit of $\mathbb{R}[x]_{\left(1+x^{2}\right)}[y]$ contradicting the indecomposability of $\alpha$ over $\mathbb{R}[x]_{\left(1+x^{2}\right)}[y]$. Since by (3.2) any quadratic space of rank $\leqslant 2$ over $\mathbb{R}[x, y]$ is extended from $\mathbb{R}$ and hence represents units, we assume now that $\gamma=\gamma_{1} \perp \gamma_{2}$, where $\gamma_{1}$ and $\gamma_{2}$ are indecomposable rank 3 spaces. Over $\mathbb{R}[x]_{\left(2+x^{2}\right)}[y]$, we have $\gamma_{1} \perp \gamma_{2} \stackrel{\sim}{\rightarrow} \beta \perp 1 \perp 1$, so that if $\gamma_{1}(v)+\gamma_{2}(w)=1$, we have by Lemma 3.1 that $\gamma_{1}(v)$ or $\gamma_{2}(w)$ is a unit.

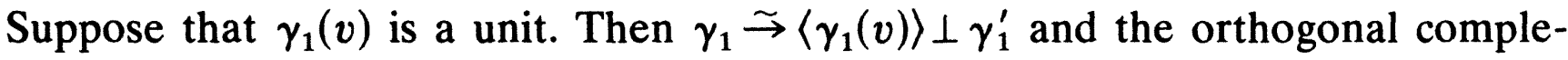
ment of $\gamma_{1}(v)+\gamma_{2}(w)$ in $\gamma_{1} \perp \gamma_{2}$ is $\gamma_{1}^{\prime} \perp \gamma_{2}^{\prime}$, where $\gamma_{2}^{\prime}$ is the orthogonal complement

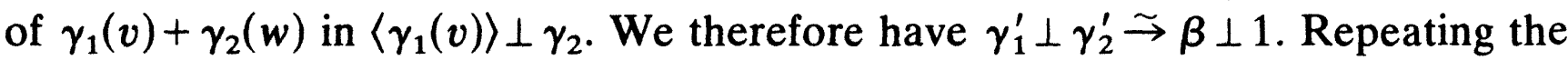
arguments over again, we get that $\beta$ is decomposable over $\mathbb{R}[x]_{\left(2+x^{2}\right)}[y]$, which is a contradiction. 


\section{REFERENCES}

[1] M. AtrYah, On the Krull-Schmidt theorem with applications to sheaves, Bull. Soc. Math. France 84, (1957), 307-317.

[2] H. BAss, Modules which support a non-singular form, J. Alg. 13, 1969, p. 246-252.

[3] H. BASS, E. H. CONNELl, and D. L. WrIGHT, Locally polynomial algebras are symmetric, Inv. Math., Vol. 38, I, 1976, p. 279-299.

[4] R. Hartshorne, Algebraic Geometry, Graduate Texts in Mathematics, 52, Springer-Verlag (1977).

[5] M.-A. Knus and M. Ojanguren, Modules and quadratic forms over polynomial algebras, Proc. Amer. Math. Soc. 66 (1977), 223-226.

[6] M.-A. Knus, M. Ojanguren and R. Sridharan, Quadratic forms and Azumaya Algebras, J. Reine Angew. Math. 303/304 (1978), 231-248.

[7] M.-A. KNUS, and R. PARIMALA, Quadratic forms associated with projective modules over quaternion algebras, J. Reine Angew. Math. 318 (1980), 20-31.

[8] M.-A. Knus, R. Parimala and R. SRidharan, Non-free projective modules over $\mathbb{H}[x, y]$ and stable bundles over $\mathbb{P}_{2}(\mathbb{C})$, Inv. Math. 65 (1981), 13-27.

[9] T. Y. LAM, Serre's Conjecture, Lecture notes in Mathematics, 635, Springer-Verlag, 1978.

[10] M. Ojanguren and R. SRIdharan, Cancellation of Azumaya algebras, J. Algebra 18 (1971), 501-505.

[11] M. Ojanguren, R. Parimala and R. SRidharan, Indecomposable quadratic bundles of rank $4 n$ over the real affine plane (Preprint).

[12] R. Okonek, M. Schneider, H. Spindler, "Vector bundles over complex projective spaces", Progress in Mathematics 3, Birkhäuser-Verlag 1980.

[13] S. PARImala, Failure of a quadratic analogue of Serre's conjecture, Amer. J. Math. 100 (1978), 913-924.

[14] S. Parimala and R. SRidharan, Projective modules over polynomial rings over division rings. J. Math. Kyoto Univ. 16 (1975), 129-148.

[15] H. G. Quebremann, W. Scharlau and M. Schulte, Quadratic and Hermitian forms in additive and abelian categories, J. Algebra, 59 (1979), 264-289.

[16] W. Scharlau, Remarks on symmetric bilinear forms over Euclidian domains (Preprint).

Eidg. Technische Hochschule

Mathematik

CH-8092 Zürich/Switzerland

Institut de Mathématiques

Université de Lausanne

CH-1015 Lausanne-Dorigny/Switzerland

School of Mathematics

Tata Institute of Fundamental Research

Bombay 400 005/India

Received March 8, 1982. 